\title{
Aplicaciones de Anestesia para Smartphones: VI parte
}

Abad Torrent $A$.

Servicio de Anestesia y Reanimación Hospital Universitari Vall d'Hebron

\section{Resumen}

Os ofrecemos una nueva versión sobre aplicaciones de Anestesia para Smartphones. Corresponden a diferentes áreas de nuestra especialidad.

En esta nueva revisión hemos encontrado aplicaciones que nos pueden ser útiles en diferentes áreas de nuestra especialidad. Para acceder a cada aplicación deberéis clicar sobre la imagen o el enlace. Hemos intentado introducir los enlaces para iTunes y Android.

\section{Introducción}

Os ofrecemos una nueva versión sobre aplicaciones de Anestesia para Smartphones. Corresponden a diferentes áreas de nuestra especialidad.

En esta nueva revisión hemos encontrado aplicaciones que nos pueden ser útiles en diferentes áreas de nuestra especialidad. Para acceder a cada aplicación deberéis clicar sobre la imagen o el enlace. Hemos intentado introducir los enlaces para iTunes y Android.

En esta nueva actualización hemos encontrado aplicaciones que nos pueden ser útiles en diferentes áreas de nuestra especialidad. Para acceder a cada aplicación deberéis clicar sobre la imagen o el enlace. Hemos intentado introducir los enlaces para iTunes y Android.

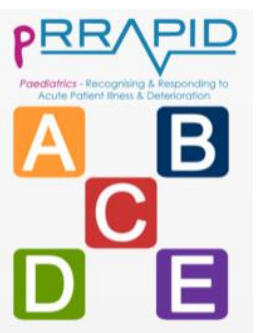

pRRAPID eBook Free

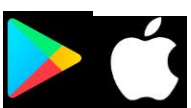

pRRAPID eBook (Paediatric Recognising and Responding to Acute Patient Illness and Deterioration)) se basa en los conceptos de RRAPID y los aplica a una población pediátrica.

La evaluación pRRAPID del niño gravemente enfermo gira en torno al enfoque tradicional basado en el sistema ABCDE. El ebook pRRAPID se lanzó en septiembre de 2016. 


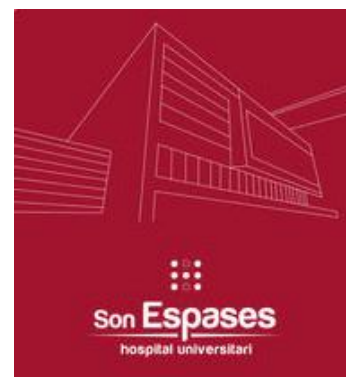

\section{Guía Terapéutica Antibiótica Free}

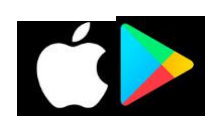

La aplicación va destinada a los médicos y farmacéuticos en general, y comprende tanto aspectos generales del uso de antimicrobianos (prevención de la aparición de resistencias, dosificación en insuficiencia renal y hepática, dosificación en pacientes obesos, farmacocinética y farmacodinámica, etc), como guías concretas de tratamiento por aparatos (respiratorio, digestivo, urinario, etc).

Hay que tener en cuenta que se trata de recomendaciones locales basadas en las situaciones específicas de nuestro centro $y$ en la información de sensibilidad antibiótica aportada por el Servicio de Microbiología del Hospital Son Espases, por lo que algunas de las recomendaciones deben ser interpretadas y adaptadas a la ubicación del lector.

También hemos querido facilitar el acceso a otros recursos como calculadoras clínicas, cursos y casos clínicos.

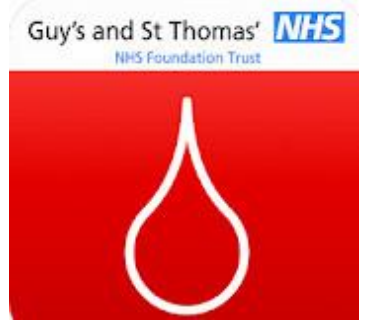

Thrombosis Guidelines Free

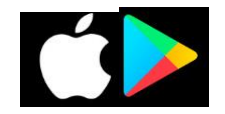

Aplicación gratuita de acceso rápido a la información para prevenir, diagnosticar y tratar la trombosis en pacientes adultos hospitalizados.

Principales características:

1) inicio de la terapia con warfarina; 2) monitorización y ajuste de la terapia con warfarina; 3) tratamiento de infusiones de heparina no fraccionada; 4) manejo de la anticoagulación y reversión de la terapia con warfarina; 5) investigación y tratamiento de trombosis venosa profunda; 5) tromboprofilaxis venosa en pacientes médicos y quirúrgicos.

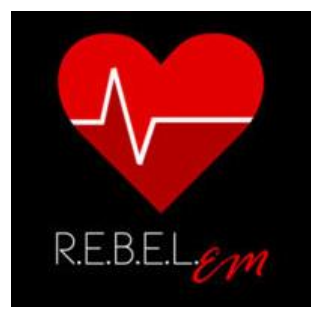

\section{$\underline{\text { R.E.B.E.L. EM Free }}$}

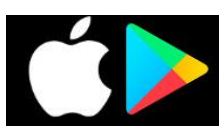

Una evaluación de la literatura de la Medicina de Emergencias basada en la evidencia científica.

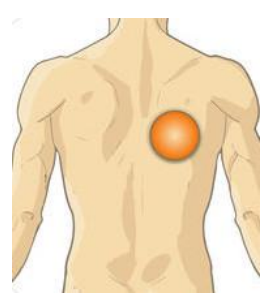

$\underline{\text { ICU Patient Communicator Free }}$

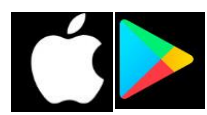


El ICU Patient Communicator de SCCM está diseñado para mejorar la comunicación entre pacientes y cuidadores. Esta aplicación permite que los pacientes que están conscientes, pero que no pueden comunicarse verbalmente, tengan la oportunidad de expresar inquietudes o problemas relacionados con el control del dolor, los niveles de comodidad y el bienestar general. Disponible en varios idiomas y traducción.

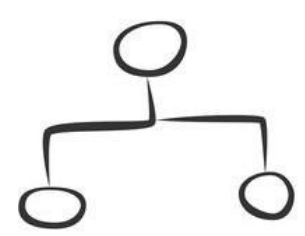

ddxof: medical algorithms Free

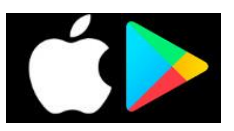

ddxof o "diagnóstico diferencial de" es una biblioteca de más de 100 algoritmos de soporte para la toma de decisiones de diagnóstico y gestión cuidadosamente seleccionados para aquellas situaciones habituales en el servicio emergencias.

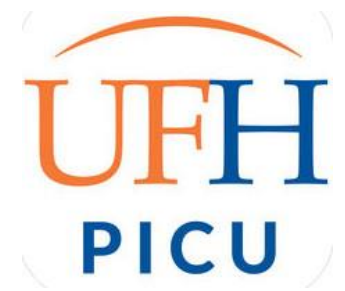

\section{$\underline{\text { PICU Essentials }}$ Free}

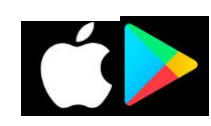

Manual práctico basado en 20 años de práctica de la UCIP, y aunque gran parte del contenido describe la práctica o los protocolos de UFHealth / Shands Children's Hospital, se considera extrapolable en la mayoría de los entornos hospitalarios.
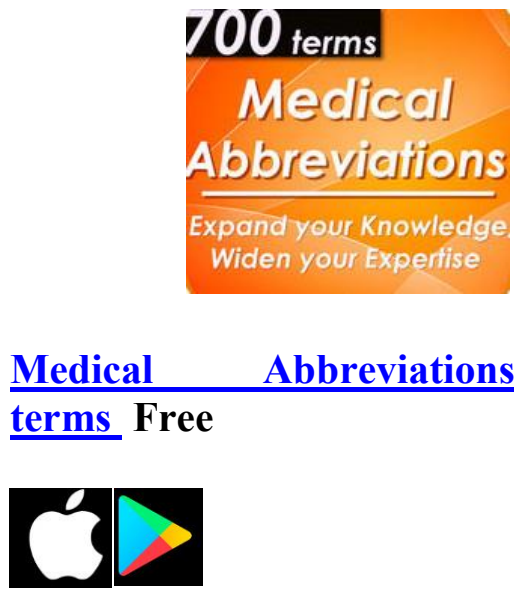

Esta es una aplicación médica profesional que contiene las últimas abreviaturas utilizadas en el campo de la medicina y que cubre la mayoría de las especialidades de medicina.

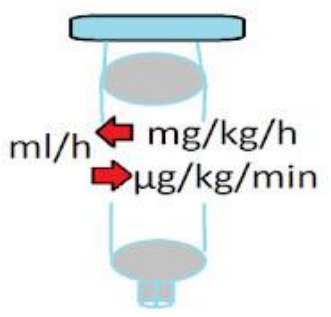
$\frac{\text { Anesthesia ICC infusion calculator }}{\underline{\mathrm{mg} / \mathrm{kg} / \mathrm{h} \mu \mathrm{g} / \mathrm{kg} / \mathrm{m}} \text { Free }}$

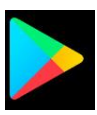

La app es un documento digital sobre el cambio de unidades usadas frecuentemente en cuidados intensivos. Con el fin de evitar errores, cuando no se introducen los parámetros necesarios la app no proporcionará ningún cálculo. Antes de su publicación ha sido testeada con profesionales sanitarios y comparada con el cálculo de bombas digitales.

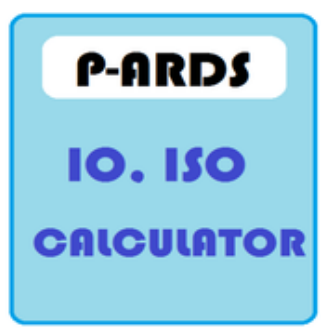


Pediatric acute respiratory distress syndrome calc Free

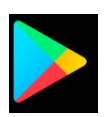

La app es un documento digital para el cálculo de la gravedad del síndrome de distres respiratorio agudo en el paciente pediátrico, en función del cálculo del índice de oxigenación o del índice de saturación de oxígeno

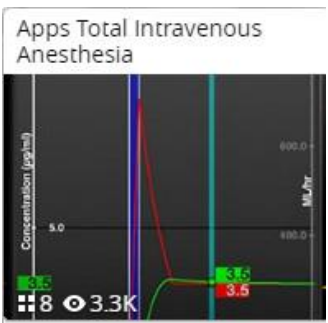

\section{Apps Total Intravenous Anesthesia}

En este archivo encontraras las aplicaciones sobre Anestesia Total Intravenosa más populares en el mercado actual. También está disponible una app para el fármaco Dexmedetomidina.

Apps de Anestesia para

Smartphones

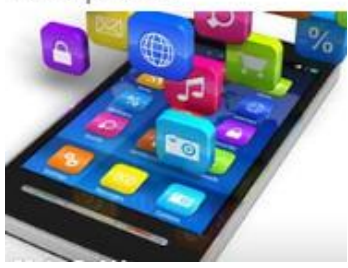

Apps de Anestesia para Smartphones

Puedes consultar las aplicaciones que recomendamos en ediciones anteriores.

Correspondencia al autor

Anna Abad Torrent aat23865@gmail.com

Servicio de Anestesia y Reanimación Hospital Universitari Vall d'Hebron

Aceptado para blog en julio de 2018. 